\title{
A QUANTITATIVE BALIAN-LOW THEOREM
}

\author{
SHAHAF NITZAN AND JAN-FREDRIK OLSEN
}

\begin{abstract}
We study functions generating Gabor Riesz bases on the integer lattice. The classical Balian-Low theorem restricts the simultaneous time and frequency localization of such functions. We obtain a quantitative estimate that extends both this result and other related theorems.
\end{abstract}

\section{INTRODUCTION}

Let $g \in L^{2}(\mathbb{R})$. The Gabor system generated by $g$ with respect to the lattice $\mathbb{Z} \times \mathbb{Z}$ is the set

$$
G(g):=\left\{\mathrm{e}^{2 \pi \mathrm{in} t} g(t-m)\right\}_{(m, n) \in \mathbb{Z}^{2}} .
$$

Lattice Gabor systems play an important role in time-frequency analysis and its applications [6, 11, 16]. A central problem in this area is to describe the optimal time-frequency localization of generators of "good" Gabor systems, e.g., orthonormal bases and Riesz bases.

The classical Balian-Low theorem [1, 5, 13] is an uncertainty principle for generators of Gabor Riesz bases. It states that if $G(g)$ is such a system, then

$$
\int_{\mathbb{R}}|g(t)|^{2} t^{2} \mathrm{~d} t=\infty \quad \text { or } \quad \int_{\mathbb{R}}|\hat{g}(\xi)|^{2} \xi^{2} \mathrm{~d} \xi=\infty .
$$

In the last 20 years, additional versions of this theorem were established. In particular we mention the $(p, q)$ Balian-Low theorem and the Amalgam BalianLow theorem (see discussion in Section 44). However, these versions do not include any quantified estimate for the decay of the generator.

A quantitative version of the classical uncertainty principle states that there exist constants $A, C>0$ such that for $g \in L^{2}(\mathbb{R})$

$$
\int_{|t| \geq R}|g(t)|^{2} \mathrm{~d} t+\int_{|\xi| \geq L}|\hat{g}(\xi)|^{2} \mathrm{~d} \xi \geq C \mathrm{e}^{-A R L}\|g\|_{L^{2}(\mathbb{R})}^{2}, \quad \forall R, L>0 .
$$

(This follows from [8]. See also Section 5.) In light of the analogy between the classical uncertainty principle and the classical Balian-Low theorem, the following question was asked by K. Grochenig: Is it possible to obtain a version of (2) for generators of Gabor Riesz bases?

Our main result is as follows.

2000 Mathematics Subject Classification. Primary 42C15; Secondary 42A38. 
Theorem 1. Let $g \in L^{2}(\mathbb{R})$. If $G(g)$ is a Riesz basis, then for $R, L \geq 1$ we have

$$
\int_{|x| \geq R}|g(t)|^{2} \mathrm{~d} t+\int_{|\xi| \geq L}|\hat{g}(\xi)|^{2} \mathrm{~d} \xi \geq \frac{C}{R L},
$$

where $C$ is a positive constant depending only on the Riesz basis bounds.

This result is sharp in the sense that, for big enough $C$, the lower bound cannot be replaced by $C \log (R L) /(R L)$.

Theorem 1 is strictly stronger than the classical Balian-Low theorem as well as its $(p, q)$ version (see Sections 4.1 and 4.2). The Amalgam Balian-Low theorem is independent of Theorem 1 (see Section 4.3). However, in Section 4.3, we find a scale of uncertainty estimates that interpolate Theorem 1 and the Amalgam Balian-Low theorem as $(2,2)$ and $(1, \infty)$ endpoints.

The structure of the paper is as follows. In Section 2, we give some needed background and prove Lemma 1, which is the main step in the proof of Theorem 1. We prove Theorem 1 in Section 3. Finally, as mentioned above, in Section 4, we discuss the connection between Theorem 1 and other Balian-Low type estimates.

\section{Preliminaries}

In this section, we recall the definitions of Riesz bases, the Zak transform, and quasi-periodic functions. Moreover, we obtain our key lemma, which quantifies the discontinuous behavior of arguments of quasi-periodic functions.

2.1. The Zak transform. The following definition provides the main tool in the study of lattice Gabor systems (see, for example, [11, Chapter 8]).

Definition 1. Let $g \in L^{2}(\mathbb{R})$. The Zak transform of $g$ is given by

$$
Z g(x, y)=\sum_{k \in \mathbb{Z}} g(x-k) \mathrm{e}^{2 \pi \mathrm{i} k y}, \quad(x, y) \in \mathbb{R}^{2} .
$$

For $g \in L^{2}(\mathbb{R})$ the function $Z g$ is quasi-periodic on $\mathbb{R}^{2}$. Namely, it satisfies

$$
Z g(x, y+1)=Z g(x, y), \quad \text { and } \quad Z g(x+1, y)=\mathrm{e}^{2 \pi \mathrm{i} y} Z g(x, y) .
$$

In particular, this implies that $Z g$ is uniquely determined by its values on $[0,1]^{2}$. It is well-known that the Zak transform induces a unitary operator from $L^{2}(\mathbb{R})$ onto $L^{2}\left([0,1]^{2}\right)$.

We will use some basic properties of the Zak transform: The Zak transform satisfies

$$
Z \hat{g}(x, y)=\mathrm{e}^{2 \pi \mathrm{i} x y} Z g(-y, x)
$$

where

$$
\hat{g}(\xi)=\int_{\mathbb{R}} g(t) \mathrm{e}^{-2 \pi \mathrm{i} \xi t} \mathrm{~d} t
$$


is the Fourier transform for functions in $L^{1}(\mathbb{R})$, which has the usual extension to functions in $L^{2}(\mathbb{R})$. Furthermore, we have

$$
Z(g * \phi)=Z g *_{x} \phi,
$$

for any Schwarz class function $\phi$, where the convolution on the right-hand side is taken with respect to the first variable.

2.2. Riesz bases. Let $H$ be a separable Hilbert space. A system $\left\{f_{n}\right\}$ in $H$ is called a Riesz basis if it is the image of an orthonormal basis under a bounded and invertible linear operator. This means that $\left\{f_{n}\right\}$ is a Riesz basis if it is complete in $H$ and

$$
A \sum\left|a_{n}\right|^{2} \leq\left\|\sum a_{n} f_{n}\right\|^{2} \leq B \sum\left|a_{n}\right|^{2}, \quad \forall\left\{a_{n}\right\} \in \ell^{2},
$$

where $A$ and $B$ are positive constants. The biggest $A$ and smallest $B$ for which (6) holds are called the Riesz basis bounds.

It is well-known that if a Gabor system

$$
G(g, a, b):=\left\{\mathrm{e}^{2 \pi \mathrm{i} b n t} g(t-m a)\right\}_{(m, n) \in \mathbb{Z}^{2}}
$$

is a Riesz basis, then $a b=1$ [15]. Although the results in this paper are stated for the case $a=b=1$, they also hold for all systems $G(g, a, b)$ with $a b=1$. This can be seen by appropriately dilating the generator function $g$.

The Zak transform allows one to characterize generators of lattice Gabor Riesz bases. Indeed, it is easy to see that $G(g)$ is such a system if and only if

$$
A \leq|Z g(x, y)|^{2} \leq B, \quad \forall(x, y) \in Q,
$$

where $A$ and $B$ are the Riesz basis bounds. This characterization implies that in order to study the discontinuous behavior of $Z g$, it is enough to understand the behavior of its argument.

2.3. Arguments of quasi-periodic functions. It is known that an argument of a quasi-periodic function can never be continuous (see, e.g., [11, Lemma 8.4.2] and the references therein). We now establish our main lemma, which is a stronger version of this statement.

First, we introduce some notation. Let $G$ be a quasi-periodic function on $\mathbb{R}^{2}$ and $H$ be a branch of its argument, i.e.,

$$
G(x, y)=|G(x, y)| \mathrm{e}^{2 \pi \mathrm{i} H(x, y)} .
$$

Fix two integers $K, N \geq 8$, and a point $(x, y) \in[0,1 / K) \times[0,1 / N)$. Set

$$
h_{i, j}=H\left(x+\frac{i}{K}, y+\frac{j}{N}\right), \quad \forall(i, j) \in \mathbb{Z}^{2} .
$$

Lemma 1. There exist two integers $0 \leq i<K$ and $0 \leq j<N$ such that either

$$
\left|h_{i+1, j}-h_{i, j}\right|>\frac{1}{8} \bmod 1
$$


or

$$
\left|h_{i, j+1}-h_{i, j}\right|>\frac{1}{8} \bmod 1
$$

Proof. Assume that both (8) and (9) do not hold, and choose a branch of the argument $H$ as follows:

- First, fix the number $h_{0,0}$ and choose $\left\{h_{i, 0}\right\}_{i=1}^{K}$ to satisfy $\left|h_{i+1,0}-h_{i, 0}\right| \leq 1 / 8$ for $0<i<K$.

- Next, for each $0 \leq i<K$, choose $\left\{h_{i, j}\right\}_{j=1}^{N}$ so that $\left|h_{i, j+1}-h_{i, j}\right| \leq 1 / 8$ for $0 \leq j<N$.

- Finally, recall that the point $(x, y)$ was fixed and use the quasi-periodicity of $G$ to observe that $h_{K, 0}=h_{0,0}+y+M$ for some integer $M$. Now, choose, for $0<j \leq N$,

$$
h_{K, j}=h_{0, j}+\frac{j}{N}+y+M .
$$

This implies that $\left|h_{K, j+1}-h_{K, j}\right| \leq 1 / 4$ for $0 \leq j<N$.

All other values of the branch $H$ may be chosen arbitrarily.

We claim that with these choices we also have

$$
\left|h_{i+1, N}-h_{i, N}\right| \leq \frac{1}{8}, \quad 0 \leq i<K .
$$

Indeed, to prove (11), first note that, since $G$ is a quasi-periodic function, this inequality holds modulo 1 . Now we may use a recursive argument: As $\mid h_{i+1,0}-$ $h_{i, 0} \mid \leq 1 / 8$, it follows, by comparing with these two terms, that $\left|h_{i+1,1}-h_{i, 1}\right| \leq 1 / 2$. But, since $\left|h_{i+1,1}-h_{i, 1}\right| \leq 1 / 8$ modulo 1 , it is clear that this inequality also holds without taking modulo 1 . Repeating this argument $N$ times, one arrives at (11).

By the periodicity of $G$ in the $y$-variable, there exist integers $L_{i}$ so that $h_{i, N}=$ $h_{i, 0}+L_{i}$. By (11), we have that $\left|L_{i+1}-L_{i}\right| \leq 1 / 4$. Hence, all $L_{i}$ are equal. In particular, $L_{K}=L_{0}$.

On the other hand, condition (10) implies that the sequence $\left\{b_{j}\right\}:=\left\{h_{K, j}-h_{0, j}\right\}$ satisfies

$$
b_{j+1}-b_{j}=\frac{1}{N}
$$

whence $L_{K}-L_{0}=b_{N}-b_{0}=1$. This gives the required contradiction.

\section{Proof of Theorem 1}

In this section, we obtain some consequences of Lemma 1 which we then use to prove our main result. 
3.1. Bounded quasi-periodic functions. A basic measure theoretic argument yields the following consequence of Lemma 1 .

Lemma 2. Fix $D>0$. There exists a positive constant $\delta=\delta(D)$ such that, given any two integers $K, N \geq 8$ and any quasi-periodic function $G$ with $|G| \geq D$ almost everywhere, one can find a set $S \subseteq[0,1]^{2}$ of measure at least $1 / N K$ such that all $(x, y) \in S$ satisfy either

$$
\left|G\left(x+\frac{1}{K}, y\right)-G(x, y)\right| \geq \delta
$$

or

$$
\left|G\left(x, y+\frac{1}{N}\right)-G(x, y)\right| \geq \delta .
$$

Next, we use Lemma 2 to evaluate the difference between the Zak transform of a function and the Zak transform of its convolution with some smooth kernel.

Lemma 3. Fix $m, M>0$. There exist positive constants $\delta=\delta(m)$ and $\eta=$ $\eta(m, M)$ such that, given any pair of Schwarz class functions $\phi, \psi$ on $\mathbb{R}$ and any $g \in L^{2}(\mathbb{R})$ with $m<|Z g|<M$ almost everywhere, one can find a set $S \subseteq[0,1]^{2}$ of measure at least $\eta /\left(1+\int\left|\phi^{\prime}\right|\right)\left(1+\int\left|\psi^{\prime}\right|\right)$ such that all $(x, y) \in S$ satisfy either

$$
|Z g(x, y)-Z(g * \phi)(x, y)| \geq \delta
$$

or

$$
|Z \hat{g}(x, y)-Z(\hat{g} * \psi)(x, y)| \geq \delta .
$$

Proof. Observe that by (5), for all $K>0$, we have

$$
|Z(g * \phi)(x+1 / K, y)-Z(g * \phi)(x, y)| \leq \frac{M}{K} \int_{\mathbb{R}}\left|\phi^{\prime}(\tau)\right| \mathrm{d} \tau .
$$

For $G=Z g$ and $D=m$, let $\delta_{1}>0$ be the constant from Lemma 2. Choose the smallest integers $N, K \geq 8$ that satisfy

$$
K \geq \frac{2 M}{\delta_{1}} \int\left|\phi^{\prime}\right| \quad \text { and } \quad N \geq \frac{4 \pi M}{\delta_{1}}\left(\int\left|\psi^{\prime}\right|+1\right),
$$

and let $S_{1}$ be the set described in Lemma 2 .

If $(x, y) \in S_{1}$ satisfies (12), then, in light of (16), we find that either $(x, y)$ or $(x+1 / K, y)$ satisfy (14) with $\delta=\delta_{1} / 4$.

On the other hand, if $(x, y) \in S_{1}$ satisfies (13), then (4) and the quasi-periodicity of $Z g$ imply that

$$
|Z \hat{g}(1-y-1 / N, x)-Z \hat{g}(1-y, x)| \geq \delta_{1}-\frac{2 \pi}{N} M \geq \frac{\delta_{1}}{2} .
$$

Applying (16) once again, we find that either $(1-y, x)$ or $(1-y-1 / N, x)$ satisfy (15) with $\delta=\delta_{1} / 8$.

In this way we find a set $S$ of measure at least $\left|S_{1}\right| / 4$ with the required properties. 
(It may happen that $(x+1 / K, y)$ or $(1-y-1 / N, x)$ fall outside of $[0,1]^{2}$. However, as the functions discussed are quasi-periodic, the conclusion remains true if $x+1 / K$ and $1-y-1 / N$ are taken modulo 1 ).

\subsection{Proof of Theorem 1 .}

Proof. Let $g \in L^{2}(\mathbb{R})$ be such that $G(g)$ is a Riesz basis with bounds $A$ and $B$. By (17), we have $m \leq|Z g| \leq M$ with $m=\sqrt{A}$ and $M=\sqrt{B}$.

Fix a Schwarz class function $\rho$ such that $\hat{\rho}$ is symmetric, satisfies $|\hat{\rho}| \leq 1$ and

$$
\hat{\rho}(\xi)=\left\{\begin{array}{lll}
1 & \text { if } \quad|\xi| \leq 1, \\
0 & \text { if } \quad|\xi| \geq 2 .
\end{array}\right.
$$

Given $R, L \geq 1$, we apply Lemma 3 with $\phi(t)=R \rho(R t)$ and $\psi(t)=L \rho(L t)$. We find that, for some constants $C=C(A, B)$ and $\delta=\delta(A, B)$, there exists a set $S \subseteq[0,1]^{2}$ with measure at least $C / R L$ such that all $(x, y) \in S$ satisfy

$$
\delta^{2} \leq|Z \hat{g}-Z(\hat{g} * \phi)|^{2}+|Z g-Z(g * \psi)|^{2} .
$$

From this and (44) it follows that

$$
\begin{aligned}
\frac{C \delta^{2}}{R L} & \leq \iint_{[0,1]^{2}}|Z \hat{g}-Z(\hat{g} * \phi)|^{2}+|Z g-Z(g * \psi)|^{2} \mathrm{~d} x \mathrm{~d} y \\
& =\iint_{[0,1]^{2}}|Z(g(1-\hat{\phi}))|^{2}+|Z(\hat{g}(1-\hat{\psi}))|^{2} \mathrm{~d} x \mathrm{~d} y .
\end{aligned}
$$

Since the Zak transform is a unitary operator from $L^{2}(\mathbb{R})$ to $L^{2}\left([0,1]^{2}\right)$, this gives

$$
\begin{aligned}
\frac{C_{1}}{R L} & \leq \int_{\mathbb{R}}|g(t)|^{2}|1-\hat{\phi}(t)|^{2} \mathrm{~d} t+\int_{\mathbb{R}}|\hat{g}(\xi)|^{2}|1-\hat{\psi}(\xi)|^{2} \mathrm{~d} \xi \\
& \leq \int_{|t|>R}|g(t)|^{2} \mathrm{~d} t+\int_{|\xi|>L}|\hat{g}(\xi)|^{2} \mathrm{~d} \xi,
\end{aligned}
$$

where $C_{1}=C \delta^{2}$ is a positive constant depending only on $A$ and $B$.

To see that the result is sharp, we use the function $g$ defined in [2, Definition 2.3]. On the one hand, this function satisfies $|Z g|=1$ for all $(x, y) \in \mathbb{R}^{2}$, and therefore the system $G(g)$ is an orthonormal basis. On the other hand, by following the proofs of Theorems 3.4 and 3.10 in the same reference, it follows that

$$
\int_{|t|>R}|g(t)|^{2} \mathrm{~d} t+\int_{|\xi|>L}|\hat{g}(\xi)|^{2} \mathrm{~d} \xi \leq \frac{1}{R^{2}}+\frac{\log L}{L^{2}} .
$$

This ends the proof.

\section{TheOREM 1 In COMPARISON TO RELATED RESUltS}

In this section, we discuss the connection between Theorem 1, the classical Balian-Low theorem and other results in this field. In particular, in Section 4.2 we study the connection to the $(p, q)$ Balian-Low theorem and in Section 4.3 the connection to the Amalgam Balian-Low theorem. 
4.1. The classical Balian-Low theorem. We begin by observing that Theorem 1 implies the Balian-Low theorem. Indeed, it is enough to notice that if $g \in L^{2}(\mathbb{R})$ satisfies (3), then for every $R>0$ we have

$$
\begin{aligned}
C & \leq R^{2} \int_{|t|>R}|g(t)|^{2} \mathrm{~d} t+R^{2} \int_{|\xi|>R}|\hat{g}(\xi)|^{2} \mathrm{~d} \xi \\
& \leq \int_{|t|>R}|t|^{2}|g(t)|^{2} \mathrm{~d} t+\int_{|\xi|>R}|\xi|^{2}|\hat{g}(\xi)|^{2} \mathrm{~d} \xi,
\end{aligned}
$$

whence at least one of the integrals in (1) diverges.

This proves the first half of the following proposition.

Proposition 1. If a function in $L^{2}(\mathbb{R})$ satisfies condition (3), then it also satisfies condition (1). Moreover, there exists a function $g \in L^{2}(\mathbb{R})$ that satisfies condition (11) but does not satisfy condition (3i).

Proof. Throughout this proof, we let $C$ denote different constants which may change from line to line. Let

$$
g(t)=\sum_{k=1}^{\infty} \frac{\sin ^{2}\left(t-2^{k}\right)}{k^{1 / 2} 2^{k}\left(t-2^{k}\right)^{2}}
$$

and note that its Fourier transform is supported on $[-2,2]$. The function $g$ satisfies

$$
\int_{\mathbb{R}}|t|^{2}|g(t)|^{2} \mathrm{~d} t \geq \sum_{k=1}^{\infty} \frac{1}{k} \int_{2^{k}}^{2^{k}+1} \frac{\sin ^{4}\left(t-2^{k}\right)}{\left(t-2^{k}\right)^{4}} \mathrm{~d} t=\infty .
$$

Given a positive integer $n$, set $R_{n}=\left[2^{n}+2^{(n+1)}\right] / 2$. To estimate

$$
I:=\int_{|t|>R_{n}}|g(t)|^{2} \mathrm{~d} t
$$

write $g=\sum_{k=1}^{n}+\sum_{k=n+1}^{\infty}$ and correspondingly $I<2 I_{1}+2 I_{2}$. By the CauchySchwartz inequality, we have

$$
I_{1}:=\int_{|t|>R_{n}}\left|\sum_{k=1}^{n} \frac{1}{k^{1 / 2} 2^{k}\left(t-2^{k}\right)^{2}}\right|^{2} \mathrm{~d} t \leq C \frac{n}{2^{3 n}},
$$

which is smaller than $C / R_{n}^{2}$ whenever $n$ is big enough. We turn to estimating $I_{2}$. To this end let $w$ be the Fourier transform of $\sin ^{2} t / t$. We have

$$
I_{2} \leq \int_{\mathbb{R}}\left|\sum_{k=n+1}^{\infty} \frac{1}{k^{1 / 2} 2^{k}} e^{i 2^{k} \xi} w(\xi)\right|^{2} \mathrm{~d} t \leq C \frac{1}{n 2^{2 n}},
$$

which is smaller than $C / R_{n}^{2}$ whenever $n$ is big enough. It follows that the function $g$ satisfies condition (1), while condition (3) is not fulfilled.

Remark 1. In fact, the function $g+\hat{g}$ is an example of a function that does not satisfy condition (3), but for which both integrals in (11) diverge. 
To better understand the relation between Theorem 1 and the classical BalianLow theorem, we consider the symmetric case $L=R$. In this case, $g \in L^{2}(\mathbb{R})$ satisfies condition (3) of Theorem 1 if and only if

$$
\liminf _{R \rightarrow \infty} R^{2}\left(\int_{|t|>R}|g(t)|^{2} \mathrm{~d} t+\int_{|\xi|>R}|\hat{g}(\xi)|^{2} \mathrm{~d} \xi\right)>C .
$$

The classical Balian-Low theorem is related to similar statements, with lim sup in place of lim inf. Indeed, the calculation (17) implies that if a function $g \in L^{2}(\mathbb{R})$ satisfies condition (18) with this modification, then it also satisfies condition (11).

As a converse to this statement, it is easy to show the following. If $g \in L^{2}(\mathbb{R})$ satisfies condition (11) then, for every $\epsilon>0$, it holds that

$$
\limsup _{R \rightarrow \infty} R^{2+\epsilon}\left(\int_{|t|>R}|g(t)|^{2} \mathrm{~d} t+\int_{|\xi|>R}|\hat{g}(\xi)|^{2} \mathrm{~d} \xi\right)=\infty .
$$

This is sharp in the sense that removing the $\epsilon$ may give a limit equal to 0 .

4.2. The $(p, q)$ Balian-Low theorem. Fix $1 \leq p \leq 2$ and let $q$ be such that $1 / p+1 / q=1$. The $(p, q)$ Balian-Low theorem states that if $g \in L^{2}(\mathbb{R})$ generates a Gabor Riesz basis on the integer lattice, then

$$
\int|t|^{p}|g(t)|^{2} \mathrm{~d} t=\infty \quad \text { or } \quad \int|\xi|^{q}|\hat{g}(\xi)|^{2} \mathrm{~d} \xi=\infty .
$$

For exponents $p+\epsilon$ and $q+\epsilon$ in place of $p$ and $q$, the original proof follows by combining [7, Theorem 4.4] with [10, Theorem 1]. This was improved to (19) in [9] using methods from the theory of VMO functions.

The following proposition implies that the $(p, q)$ Balian-Low theorem follows from Theorem 1 .

Proposition 2. If a function in $L^{2}(\mathbb{R})$ satisfies condition (3), then it also satisfies condition (19). Moreover, there exists a function $g \in L^{2}(\mathbb{R})$ that satisfies condition (19), but does not satisfy condition (3).

Proof. Assume that $g \in L^{2}(\mathbb{R})$ satisfies condition (3). For $R>0$ and $L=R^{p-1}$, this implies that

$$
C \leq \int_{|t|>R}|t|^{p}|g(t)|^{2} \mathrm{~d} t+\int_{|\xi|>R^{p-1}}|\xi|^{q}|\hat{g}(\xi)|^{2} \mathrm{~d} \xi,
$$

which means that at least one of the integrals in (19) diverges.

The second part of the statement follows from Remark 1.

4.3. The Amalgam Balian-Low theorem. The Amalgam Balian-Low Theorem [12, Corollary 7.5.3] states that if $g \in L^{2}(\mathbb{R})$ generates a Gabor Riesz Basis on the integer lattice, then

$$
\sum_{n}\|g(t)\|_{L^{\infty}[n, n+1]}=\infty \quad \text { or } \quad \sum_{n}\|\hat{g}(\xi)\|_{L^{\infty}[n, n+1]}=\infty .
$$


This theorem is known to be independent of the classical Balian-Low theorem 3 , Examples 3.3 and 3.4]. A simple calculation shows that the same examples also yield that the Amalgam Balian-Low theorem is independent of Theorem 1;

Proposition 3. There exists a function $g \in L^{2}(\mathbb{R})$ that satisfies condition (3) but does not satisfy condition (20). On the other hand, there exists a function $g \in L^{2}(\mathbb{R})$ that satisfies condition (20), but does not satisfy condition (3).

We proceed to show that the Amalgam Balian-Low theorem and Theorem 1 are end-points of a more general Balian-Low type theorem.

Theorem 2. Let $1 \leq p \leq 2$ and $q$ be such that $1 / p+1 / q=1$. If $G(g)$ is a Riesz basis, then for $R, L \geq 1$ we have

$$
\frac{C}{(R L)^{p / q}}<\sum_{|k|>R}\|f\|_{L^{q}(k, k+1)}^{p}+\sum_{|k|>L}\|\hat{f}\|_{L^{q}(k, k+1)}^{p},
$$

where $C$ is a positive constant depending only on the Riesz basis bounds.

Note that for $p=q=2$ we get Theorem 1, while for $p=1$ and $q=\infty$ we get the Amalgam Balian-Low theorem.

Proof. It is easy to verify that the Zak transform satisfies

$$
\|Z g\|_{L^{2}(Q)}^{2}=\|g\|_{L^{2}(\mathbb{R})}^{2}=\sum_{k}\|g\|_{L^{2}(k, k+1)}^{2},
$$

and

$$
\|Z g\|_{L^{\infty}(Q)} \leq \sum_{k}\|g\|_{L^{\infty}(k, k+1)}
$$

By the method of complex interpolation for vector valued sequence spaces (see, e.g., [4, Theorem 5.1.2]), this implies that

$$
\|Z g\|_{L^{q}(Q)}^{p} \leq \sum_{k}\|g\|_{L^{q}(k, k+1)}^{p}
$$

We first prove the theorem for $1<p<2$. Following the same technique as in the proof of Theorem 1, we use Lemma 3 to find that

$$
\begin{aligned}
\frac{C \delta^{q}}{R L} & \leq \iint_{Q}|Z \hat{g}-Z(\hat{g} * \phi)|^{q}+|Z g-Z(g * \psi)|^{q} \mathrm{~d} x \mathrm{~d} y \\
& =\iint_{Q}|Z(g(1-\hat{\phi}))|^{q}+|Z(\hat{g}(1-\hat{\psi}))|^{q} \mathrm{~d} x \mathrm{~d} y .
\end{aligned}
$$

By (21), this is smaller than

$$
\left(\sum_{k}\|g(1-\hat{\phi})\|_{L^{q}(k, k+1)}^{p}\right)^{q / p}+\left(\sum_{k}\|\hat{g}(1-\hat{\psi})\|_{L^{q}(k, k+1)}^{p}\right)^{q / p}
$$




$$
\leq\left(\sum_{|k|>R}\|g\|_{L^{q}(k, k+1)}^{p}\right)^{q / p}+\left(\sum_{|k|>L}\|\hat{g}\|_{L^{q}(k, k+1)}^{p}\right)^{q / p}
$$

The desired inequality now follows from the fact that $|x|^{\beta}+|y|^{\beta} \leq C(|x|+|y|)^{\beta}$ for all $\beta \geq 0$, with $C>0$ depending only on $\beta$.

The case $p=1$ is proved in much the same way.

\section{OPEN PROBLEM}

A much more general version of (2) was obtained by F. Nazarov [14]: There exist constants $A, C>0$ such that for $g \in L^{2}(\mathbb{R})$ and any two sets $S, K \subset \mathbb{R}$ of finite measure the following inequality holds

$$
\int_{\mathbb{R} \backslash S}|g(x)|^{2} \mathrm{~d} x+\int_{\mathbb{R} \backslash K}|\hat{g}(\xi)|^{2} \mathrm{~d} \xi \geq C \mathrm{e}^{-A|S||K|}\|g\|_{L^{2}(\mathbb{R})}^{2} .
$$

Does this theorem have a version for functions $g$ that generate Gabor Riesz bases on the integer lattice?

\section{ACKNOWLEDGEMENTS}

The authors wish to thank A. Aleman for inviting the first author to Lund university and M. Sodin for inviting the second author to Tel-Aviv university. These visits provided us with the best setting possible for completing this project.

\section{REFERENCES}

1. Roger Balian, Un principe d'incertitude fort en théorie du signal ou en mécanique quantique, C. R. Acad. Sci. Paris Sér. II Méc. Phys. Chim. Sci. Univers Sci. Terre 292 (1981), no. 20, 1357-1362.

2. John J. Benedetto, Wojciech Czaja, Przemyslaw Gadziński, and Alexander M. Powell, The Balian-Low theorem and regularity of Gabor systems, J. Geom. Anal. 13 (2003), no. 2, 239-254.

3. John J. Benedetto, Christopher Heil, and David F. Walnut, Differentiation and the BalianLow theorem, J. Fourier Anal. Appl. 1 (1995), no. 4, 355-402.

4. Jöran Bergh and Jörgen Löfström, Interpolation spaces. An introduction, Springer-Verlag, Berlin, 1976, Grundlehren der Mathematischen Wissenschaften, No. 223.

5. Ingrid Daubechies, The wavelet transform, time-frequency localization and signal analysis, IEEE Trans. Inform. Theory 36 (1990), no. 5, 961-1005.

6. Monika Dörfler, Time-frequency analysis for music signals: A mathematical approach, J. New Mus. Res. 30 (2001), no. 1, 3-12.

7. Hans G. Feichtinger and K. Gröchenig, Gabor frames and time-frequency analysis of distributions, J. Funct. Anal. 146 (1997), no. 2, 464-495.

8. W. H. J. Fuchs, On the eigenvalues of an integral equation arising in the theory of bandlimited signals, J. Math. Anal. Appl. 9 (1964), 317-330.

9. Sushrut Z. Gautam, A critical-exponent Balian-Low theorem, Math. Res. Lett. 15 (2008), no. 3, 471-483.

10. Karlheinz Gröchenig, An uncertainty principle related to the Poisson summation formula, Studia Math. 121 (1996), no. 1, 87-104. 
11. Birkhäuser Boston Inc., Boston, MA, 2001.

12. Christopher Edward Heil, Wiener amalgam spaces in generalized harmonic analysis and wavelet theory, ProQuest LLC, Ann Arbor, MI, 1990, Thesis (Ph.D.)-University of Maryland, College Park.

13. Francis E. Low, Complete sets of wave packets, A passion for physics - essays in honor of Geoffrey Chew (C. DeTar et al., ed.), World Scientific, Singapore, 1985, pp. 17-22.

14. F. L. Nazarov, Local estimates for exponential polynomials and their applications to inequalities of the uncertainty principle type, Algebra i Analiz 5 (1993), no. 4, 3-66.

15. Jayakumar Ramanathan and Tim Steger, Incompleteness of sparse coherent states, Appl. Comput. Harmon. Anal. 2 (1995), no. 2, 148-153.

16. Thomas Strohmer, Pseudodifferential operators and Banach algebras in mobile communications, Appl. Comput. Harmon. Anal. 20 (2006), no. 2, 237-249.

School of Mathematical Sciences, Weizmann Institute for Science, Rehovot 76100 , ISRAEL.

E-mail address: shahaf.nitzansi@weizmann.ac.il

Centre for Mathematical Sciences, Lund University, P.O. Box 118, SE-221 00 Lund, Sweden

E-mail address: janfreol@maths.1th.se 